\title{
Intrinsic colour calibration for F, G, K stars
}

\author{
P. Bonifacio ${ }^{1}$, E. Caffau ${ }^{2}$, and P. Molaro ${ }^{1}$ \\ 1 Osservatorio Astronomico di Trieste, Via G.B. Tiepolo 11, I-34131 Trieste, Italy \\ 2 Istituto Magistrale S.P.P. e L. annesso al Convitto Nazionale Paolo Diacono, S. Pietro al Nat. Udine, Italy
}

Received May 5; accepted June 29, 2000

\begin{abstract}
We derive an intrinsic colour calibration for $\mathrm{F}-$ $\mathrm{K}$ stars using broad band Johnson colours and line indices $K P$ and $H P 2$. Through this calibration we can determine $E(B-V)$ of an individual star within $0.03 \mathrm{mag}$. The $E(B-V)$ values thus derived are in excellent agreement with those derived from Strömgren photometry through the Schuster \& Nissen (1989) calibration. The agreement is also good with the reddening maps of Burstein \& Heiles (1982) and Schlegel et al. (1998), although in this case there exists a small offset of about $0.01 \mathrm{mag}$. This calibration may be applied to the large body of data of the HK survey extension which will be published in the near future.
\end{abstract}

Key words: stars: atmospheres — stars: general — ISM: dust, extinction

\section{Introduction}

An intrinsic colour calibration is a relation defining, either in some colour-colour plane, or in some $N$-dimensional space if the calibration requires $N$ colour indices, the locus occupied by stars. The calibration is called "intrinsic" because it holds for the colours which emerge from the stellar atmosphere but not for the observed colours, which are altered while passing through the interstellar medium. Knowledge of an intrinsic colour calibration allows one, at least in principle, to derive the reddening of a given star by comparing the observed colours with the colours predicted by the calibration. This may fail if the effect of reddening is to shift the point representative of a star along the curve, i.e. if the reddening vector is parallel to the intrinsic locus. This occurs for instance in the $((B-V),(U-B))$ plane for $\mathrm{F}, \mathrm{G}, \mathrm{K}$ stars where the reddening vector in the $((B-V),(U-B))$ plane is almost parallel to the intrinsic locus of Main Sequence stars.

Send offprint requests to: P. Bonifacio

e-mail: bonifaci@ts.astro.it
Determining the reddening of an individual star is important for several reasons. First, estimation of reddening is necessary if we want to obtain a photometric distance of the star. Second, it is necessary for the derivation of atmospheric parameters of the star, such as effective temperature, surface gravity and metallicity. These parameters can be derived from suitable colours, provided the reddening is properly taken into account. For example the $(V-K)$ colour may be accurately calibrated onto $T_{\text {eff }}$ (Alonso et al. 1996, 1999; Di Benedetto 1998), but using $(V-K)$ rather than $(V-K)_{0}$ will result in an underestimate of the temperature. Finally the knowledge of the reddening gives important information on the interstellar medium along the line of sight towards the star.

For F, G and $\mathrm{K}$ stars the calibration of uvby $\beta$ colours by Schuster \& Nissen (1989) has proved to be both a very powerful and accurate tool for estimating the reddening and has become the "standard" procedure for its determination. The possibility of deriving such a calibration was foreseen in the very design of the Strömgren photometric system and was already exploited by the calibrations of Crawford (1975) and Olsen (1983) which preceded the Schuster \& Nissen calibration and are superseded by it. The calibration is possible because the system provides two indices, $(b-y)$ and $\beta$, which mainly depend on effective temperature; however while $(b-y)$ depends on reddening the $\beta$ index is reddening-independent. Therefore there exists a functional relation $(b-y)_{0}=f(\beta)$ which allows us to calibrate the reddening of the observed $(b-y)$. This is accomplished by the Schuster \& Nissen calibration in which terms in $m 1$ allow us to take into account the metallicity dependence of $(b-y)$, while terms in $c 1$ allow us to take into account its dependence on surface gravity (luminosity). Although very powerful, Strömgren photometry requires a considerable investment in telescope time, due to the large number of filters (6) and to their relatively narrow width.

The HK objective-prism/interference filter survey (Beers et al. 1985, 1992) provides, at present, the largest sample of stars suited for the study of the galactic 
structure. The survey is kinematically unbiased and therefore it is ideal for studying both kinematics and dynamics of the Galactic Halo. Besides being the main source of extremely metal-poor stars, $[\mathrm{Fe} / \mathrm{H}]<-3.0$, it provides a large number of stars in the range $-0.5 \leq[\mathrm{Fe} / \mathrm{H}] \leq-2.0$ which are well suited to study both the thick-disc and the halo thick-disc transition. The medium dispersion follow-up survey, which provides radial velocities and metallicities, has been extended by Beers and collaborators in both northern and southern hemispheres and the results will be soon available (see Beers 1999 for a summary). At the same time photometric campaigns are being carried out to complement spectroscopic data. Norris et al. (1999) provide $U B V$ data for $\sim 2500$ stars, Preston et al. (1991) for about 1800 stars, Doinidis \& Beers $(1990,1991)$ for about 300 stars and Bonifacio et al. (2000) for about 300 stars. Strömgren photometry is provided for 89 stars by Schuster et al. (1996), and for $\sim 500$ stars by Anthony-Twarog et al. (2000), although the latter data do not include the $\beta$ index and therefore cannot be used to derive reddenings from the Schuster \& Nissen calibration.

From the above summary it is clear that the Schuster \& Nissen calibration is of little use in determining reddenings for HK stars and would require further observational efforts to obtain also uvby $\beta$ data. So far reddenings for these stars have been determined from maps, those of Burstein \& Heiles (1982) in the first place, and, more recently, those of Schlegel et al. (1998). However it is possible to determine reddenings from available data by developing a suitable Schuster \& Nissen - type calibration. The indices involved in the Schuster \& Nissen calibration are mostly measures of the following quantities: slope of the Paschen continuum $(b-y)$, metallicity $(m 1)$, Balmer jump $(c 1), \mathrm{H} \beta(\beta)$. Johnson photometry provides the slope of the Paschen continuum $(B-V)$ and the Balmer jump $(U-B)$, the line index $K P$ defined in Beers et al. (1999) is sensitive to metallicity, while the index $H P 2$ is a pseudoequivalent-width of $\mathrm{H} \delta$. It is therefore reasonable to expect that a Schuster \& Nissen - type calibration, involving $(B-V),(U-B), H P 2$ and $K P$ may be derived. In the following we show that this is indeed the case and that reddening may be derived from it with an accuracy comparable to that of the Schuster \& Nissen calibration.

\section{Derivation of the calibration}

To derive the calibration we used the sample of stars used by Beers et al. (1999) for the calibration of the $K P$ index. For all these stars $U B V$ photometry and $H P 2$ and $K P$ indices are available. As calibrators we selected the stars with low reddening from Beers et al. (i.e. $E(B-V) \leq$ $0.01)$. To make this criterion more stringent and thus to select a sample of truly unreddened stars, we searched the Hauck \& Mermilliod (1998) uvby $\beta$ catalog, through the interface of the General Catalog of Photometric Data (GCPD, Mermilliod et al. 1996) to find out stars of the Beers et al. sample with uvby $\beta$ photometry. We then applied the Schuster \& Nissen calibration to these data to obtain $E(b-y) \sim 0.74 E(B-V)$. Our final criterion was that both the estimate of $E(B-V)$ of Beers et al. and the one derived from Strömgren photometry were less than or equal to $0.01 \mathrm{mag}^{1}$.

Out of the sample of Beers et al. (1999) 65 stars satisfy our criterion and they are reported in Table 1 , the star name is given in Col. (1), Col. (2) gives the star type, according to Beers et al. (1999). Column (3) is $[\mathrm{Fe} / \mathrm{H}]$ and Cols. (4)-(7) provide the Strömgren photometry extracted from the Hauck \& Mermilliod (1998) catalogue. We performed a $\chi^{2}$ fit on this sample of stars for several functional forms. We computed the rms of the fit and we then discarded those stars whose residual was greater than $2.5 \times$ rms. This was aimed at further cleaning the sample by rejecting stars which are either reddened or whose colours or line indices are affected by larger errors. Quite interesting only one star, CD $-24^{\circ} 17504$, was discarded, whichever functional form was used. This star is also the most metal-poor of the sample, in fact the only one below $[\mathrm{Fe} / \mathrm{H}]=-3.00$. Thus our fits were all performed on a sample totalling 64 stars.

We began with the assumption that both $(B-V)$ and $H P 2$ strongly depend on temperature; we therefore fit a linear relation

\section{$(B-V)=x_{1}+x_{2} \log H P 2$}

and obtained a fit with $\chi^{2}=273.8$. We next began to add other terms in $\log K P$ and $(U-B)$ and checked the significance of the new term through the $\mathrm{F}$ test (Bevington \& Robinson 1992, p. 208). Linear terms in both $\log K P$ and $(U-B)$ are highly significant (the probability corresponding to the observed $F_{\chi}$ is $6.510^{-7}$ for $\log K P$ and $7.010^{-13}$ for $\left.(U-B)\right)$. On the other hand further quadratic terms are not significant, the most significant being a term in $(\log K P)^{2}$, with a probability of the observed $F_{\chi} \approx 5.510^{-2}$, i.e. a significance of $\sim 95 \%$. We therefore conclude that the best functional form is that with only linear terms.

$(B-V)_{0}=x_{1}+x_{2} \log H P 2+x_{3} \log K P+x_{4}(U-B)_{0}$.

Our best fit parameters are given in Table 2, together with their formal errors derived from the covariance matrix.

The rms of the fit was $0.0153 \mathrm{mag}$ and $\chi^{2} \approx 67.7$, i.e. $\chi_{60}^{2}=1.13$, which indicates a good fit. In Fig. 1, panel a) we show a plot of $(B-V)$ versus the right hand side of Eq. (1). Panels b) and c) display the residuals as a function of metallicity and $(B-V)$ colour, respectively.

\footnotetext{
1 Since the Schuster \& Nissen calibration may result also in negative values of $E(b-y)$ our criterion was $|1.35 E(b-y)| \leq 0.01$.
} 
Table 1. Basic data for the calibrators

\begin{tabular}{|c|c|c|c|c|c|c|}
\hline STAR & Type $^{a}$ & $\mathrm{Fe} / \mathrm{H}$ & $(b-y)$ & $m 1$ & $c 1$ & \\
\hline $\mathrm{BD}+18^{\circ} 1479$ & $\mathrm{D}$ & -0.46 & 0.440 & 0.258 & 0.256 & 2.560 \\
\hline $\mathrm{CD}-24^{\circ} 17504^{b}$ & $\mathrm{D}$ & -3.70 & 0.317 & 0.039 & 0.301 & 2.596 \\
\hline G8-16 & D & -1.59 & 0.322 & 0.071 & 0.292 & 2.595 \\
\hline $\mathrm{G} 11-36$ & $\mathrm{D}$ & -0.68 & 0.384 & 0.139 & 0.255 & 2.579 \\
\hline $11-44$ & $\mathrm{D}$ & -2.07 & 0.334 & 0.054 & 0.263 & 2.597 \\
\hline-45 & $\mathrm{D}$ & -0.01 & 0.431 & 0.249 & 0.359 & 2.602 \\
\hline $\mathrm{G} 12-21$ & $\mathrm{D}$ & -1.32 & 0.339 & 0.090 & 0.284 & 2.592 \\
\hline $\mathrm{G} 13$ & $\mathrm{D}$ & -1.63 & 0.331 & 0.060 & 0.285 & 2.594 \\
\hline & $\mathrm{D}$ & -0.27 & 0.370 & 0.150 & 0.316 & 2.590 \\
\hline $\mathrm{G} 14-26$ & $\mathrm{D}$ & -0.20 & 0.387 & 0.181 & 0.349 & 2.590 \\
\hline G15 & $\mathrm{D}$ & -0.65 & 0.436 & 0.210 & 0.253 & 2.561 \\
\hline & $\mathrm{D}$ & -0.39 & 0.475 & 0.315 & 0.246 & 2.553 \\
\hline & $\mathrm{D}$ & -0.66 & 0.367 & 0.125 & 0.306 & 2.588 \\
\hline $\mathrm{G} 17-30$ & $\mathrm{D}$ & -0.48 & 0.396 & 0.164 & 0.303 & 2.574 \\
\hline $\mathrm{G} 24$ & D & -1.10 & 0.342 & 0.092 & 0.273 & 2.597 \\
\hline & D & -1.93 & 0.351 & 0.058 & 0.208 & 2.584 \\
\hline$-\overline{6}$ & $\mathrm{D}$ & -0.54 & 0.397 & 0.160 & 0.259 & 2.566 \\
\hline 30 & $\mathrm{D}$ & -0.89 & 0.427 & 0.177 & 0.200 & 2.557 \\
\hline & $\mathrm{D}$ & -0.03 & 0.387 & 0.195 & 0.319 & 2.602 \\
\hline & $\mathrm{D}$ & 0.03 & 0.412 & 0.230 & 0.328 & 2.593 \\
\hline G58-23 & $\mathrm{D}$ & -0.97 & 0.407 & 0.138 & 0.219 & 2.565 \\
\hline & D & -1.41 & 0.344 & 0.079 & 0.258 & 2.589 \\
\hline & $\mathrm{D}$ & -0.33 & 0.371 & 0.150 & 0.344 & 2.591 \\
\hline & $\mathrm{D}$ & -1.02 & 0.424 & 0.186 & 0.204 & 2.567 \\
\hline & $\mathrm{D}$ & $-2.4 \overline{2}$ & 0.332 & 0.054 & 0.225 & 2.593 \\
\hline & D & -1.63 & 0.365 & 0.074 & 0.189 & 2.583 \\
\hline & D & -0.58 & 0.473 & 0.295 & 0.248 & 2.554 \\
\hline-52 & $\mathrm{D}$ & -1.28 & 0.430 & 0.167 & 0.190 & 2.553 \\
\hline & $\mathrm{D}$ & -0.91 & 382 & 0.139 & 0.283 & 2.584 \\
\hline & $\mathrm{D}$ & -0.35 & 0.405 & 0.188 & 0.281 & 2.584 \\
\hline & $\mathrm{D}$ & -0.20 & 0.405 & 0.204 & 0.319 & 2.582 \\
\hline & $\mathrm{D}$ & -0.78 & 0.365 & 0.126 & 0.272 & 2.586 \\
\hline & D & -2.36 & 0.309 & 0.051 & 0.357 & 2.591 \\
\hline-43 & D & -0.49 & 0.463 & 0.256 & 0.277 & 2.554 \\
\hline $3-24$ & $\mathrm{D}$ & -0.49 & 0.383 & 0.146 & 0.301 & 2.588 \\
\hline $4-\overline{2} 6$ & D & -1.78 & 0.349 & 0.076 & & 94 \\
\hline & $\mathrm{D}$ & -1.42 & 19 & 0.073 & 19 & 2.600 \\
\hline-12 & $\mathrm{D}$ & -0.92 & 0.350 & 0.094 & 0.287 & 2.592 \\
\hline G16 & $\mathrm{D}$ & -0.14 & & 0.217 & & 2.584 \\
\hline-16 & $\mathrm{D}$ & -0.53 & 0.396 & 0.167 & 23 & 2.5 \\
\hline-51 & $\mathrm{D}$ & -0.52 & 0.377 & 0.145 & & 2.5 \\
\hline G162-68 & $\mathrm{D}$ & -0.54 & & 0.202 & 06 & 2.565 \\
\hline G16 & D & -2.05 & & 0.055 & & \\
\hline & $\mathrm{D}$ & -0.45 & 0.479 & 0.304 & 0.2 & 2.5 \\
\hline & $\mathrm{D}$ & -0.50 & 0.403 & 0.190 & 0.334 & 2.579 \\
\hline G271-34 & $\mathrm{D}$ & -0.68 & & & 62 & 2.576 \\
\hline & TO & -0.38 & & 0.130 & & \\
\hline & $\overline{\mathrm{SG}}$ & -1.29 & & 0.089 & & 2.600 \\
\hline & $\mathrm{G}$ & -0.93 & & 0.199 & 34 & 2.554 \\
\hline & D & -1.71 & & 0.069 & & 2.6 \\
\hline & SG & -0.27 & 0.339 & 0.156 & & 2.0 \\
\hline & $\mathrm{D}$ & -1.61 & & 0.063 & & 2.569 \\
\hline & D & -0.99 & & 0.119 & 0.298 & 2.584 \\
\hline & & -0.2 & & & & 2.578 \\
\hline & & -0.1 & & & 0.321 & 2.591 \\
\hline $\mathrm{HD}$ & $\mathrm{D}$ & -1.04 & 0.367 & 0.122 & 0.254 & 2.587 \\
\hline & $\bar{D}$ & -0.7 & & & & 2.5 \\
\hline & $\mathrm{SC}$ & -0. & & 0.119 & & \\
\hline & $\mathrm{D}$ & -0.58 & 0.390 & 0.143 & 0.314 & 2.578 \\
\hline & $\mathrm{D}$ & -1.0 & & & 0.217 & 2.573 \\
\hline & $\mathrm{D}$ & -0.7 & & & & 2.599 \\
\hline & $\mathrm{D}$ & -0.92 & & 0.148 & & 2.577 \\
\hline HD & $\mathrm{D}$ & $-1.1 \overline{3}$ & 0.358 & 0.104 & 0.262 & 2.590 \\
\hline HD & $\overline{\mathrm{D}}$ & -0.57 & & 0.161 & 0.256 & 2.573 \\
\hline HD 2196 & $\mathrm{D}$ & -1.31 & 0.344 & 0.078 & 0.246 & 2.597 \\
\hline
\end{tabular}

${ }^{a} \mathrm{D}=$ dwarf; $\mathrm{TO}=$ turn-off; $\mathrm{SG}=$ subgiant; $\mathrm{G}=$ giant; $\mathrm{HB}=$ horizontal branch.

${ }^{b}$ Not used in the calibration, because trimmed out after the first pass.

Table 2. Best fit parameters

\begin{tabular}{rrr}
\hline & param. & error \\
\hline$x_{1}$ & 0.5734 & 0.0004 \\
$x_{2}$ & -0.2759 & 0.0006 \\
$x_{3}$ & 0.1040 & 0.0003 \\
$x_{4}$ & 0.2676 & 0.0006 \\
\hline
\end{tabular}

The range of validity of the calibration is fixed by the properties of the calibrator stars. In our case these are:

$$
\begin{aligned}
0.375 & \leq(B-V) \leq 0.800 \\
0.780 & \leq H P 2 \leq 4.330 \\
0.186 & \leq K P \\
-0.245 & \leq(U-B) \leq 0.790 \\
& \leq 0.350 .
\end{aligned}
$$

The mean metallicity of the calibrators is $[\mathrm{Fe} / \mathrm{H}] \sim-0.9$. The metallicity range is $-2.42 \leq[\mathrm{Fe} / \mathrm{H}] \leq+0.03$. In practice we expect that our calibration is applicable to stars of intermediate metallicity. We note that the mean metallicity of the calibrators of Schuster \& Nissen is $[\mathrm{Fe} / \mathrm{H}]=-0.50$, while their metallicity range is $-2.49 \leq$ $[\mathrm{Fe} / \mathrm{H}] \leq+0.22$. Thus there is an almost perfect overlap of the metallicity domains where the two calibrations are derived, with the Schuster \& Nissen calibration extending towards slightly higher metallicities.

A matter of concern is wether the luminosity (gravity) dependence of the calibration is properly captured by the $(U-B)$ colour. Inspection of Table 1 reveals that, although we did not impose any selection criterion on luminosity, most of our stars are dwarfs, only one is a giant, three sub-giants and one horizontal branch. This is a result of imposing a very tight criterion on reddening for the calibrators: all low-reddening stars are nearby and therefore most are dwarfs. So formally our calibration is valid only for dwarfs. In Fig. 2 we show the residuals of the fit as a box plot in the $\left((b-y)_{0}, c 0\right)$ plane. There appears to be no obvious trend of the residuals with the luminosity of the stars, we may therefore expect that the calibration is in fact equally applicable to dwarfs and giants, as is the Schuster \& Nissen calibration.

\section{Reddening estimation and comparison with other estimates}

Equation (1) can be used to determine $E(B-V)$ once all the observed indices are known. Since it is a first order equation both in $(B-V)$ and $(U-B)$ we may solve analytically for $E(B-V)$, rather than by iteration, as it is necessary with the Schuster \& Nissen calibration. By adopting the reddening slope $E(U-B) / E(B-V)=0.72$ we obtain:

$$
\begin{aligned}
& E(B-V)= \\
& \frac{(B-V)-x_{1}-x_{2} \log H P 2-x_{3} \log K P-x_{4}(U-B)}{\left(1-0.72 x_{4}\right)} .
\end{aligned}
$$

We can now use Eq. (2) to compute $E(B-V)$ for stars for which independent estimates of reddening are available, in order to assess the external error in the derived reddening. The internal error is of the order of the rms of the calibration, $0.015 \mathrm{mag}$.

The Beers et al. (1999) sample may be conveniently used for comparison. From the sample we exclude all 

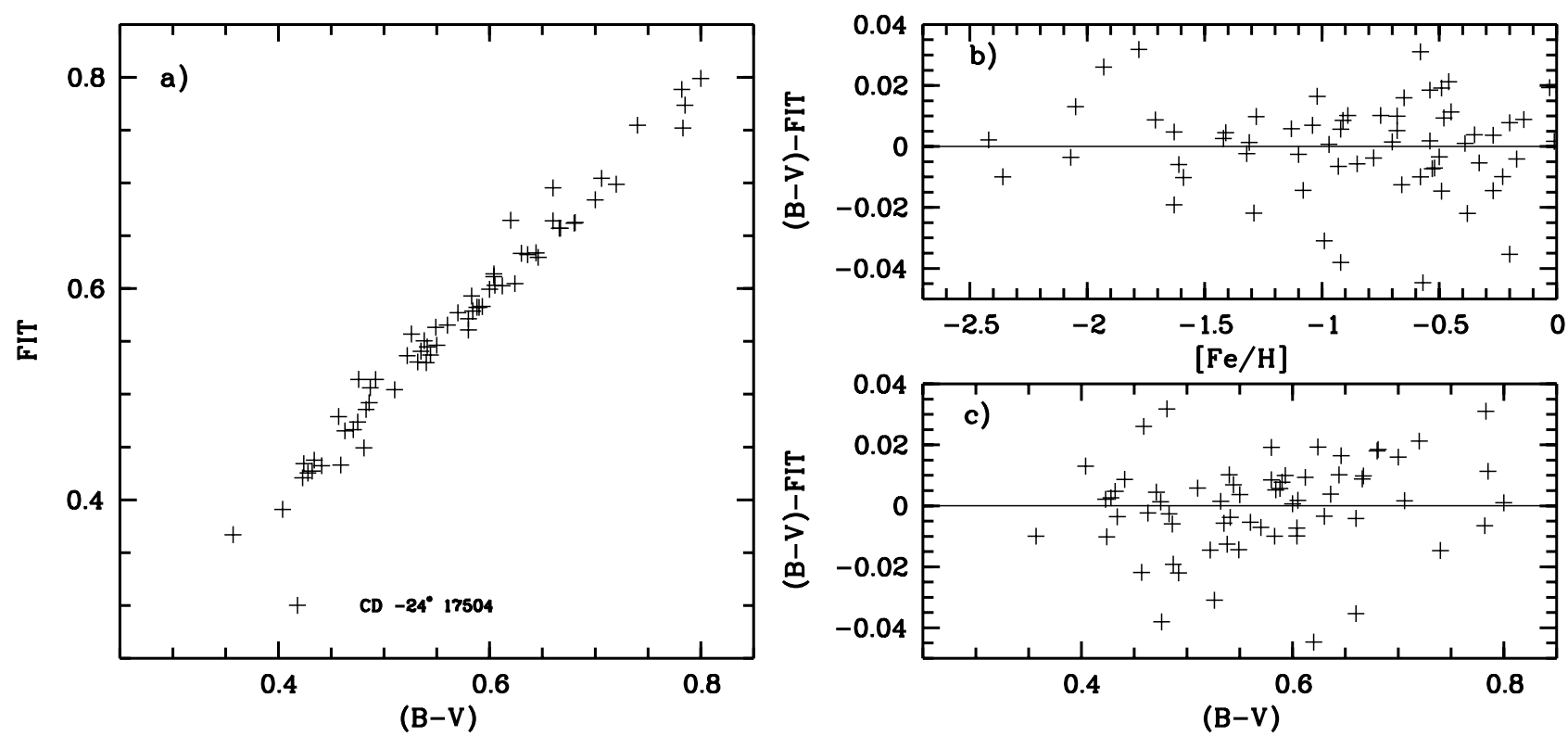

Fig. 1. a) The right hand side of Eq. (1) for the calibrators as a function of $(B-V)$. The star CD $-24^{\circ} 17504$ appears to be an outlier and has not been used in the derivation of the calibration, and it is not drawn in panels b) and c), b) the residuals $(B-V)$-FIT as a function of metallicity, $\mathbf{c})$ same as panel $\mathbf{b})$ but as a function of $(B-V)$
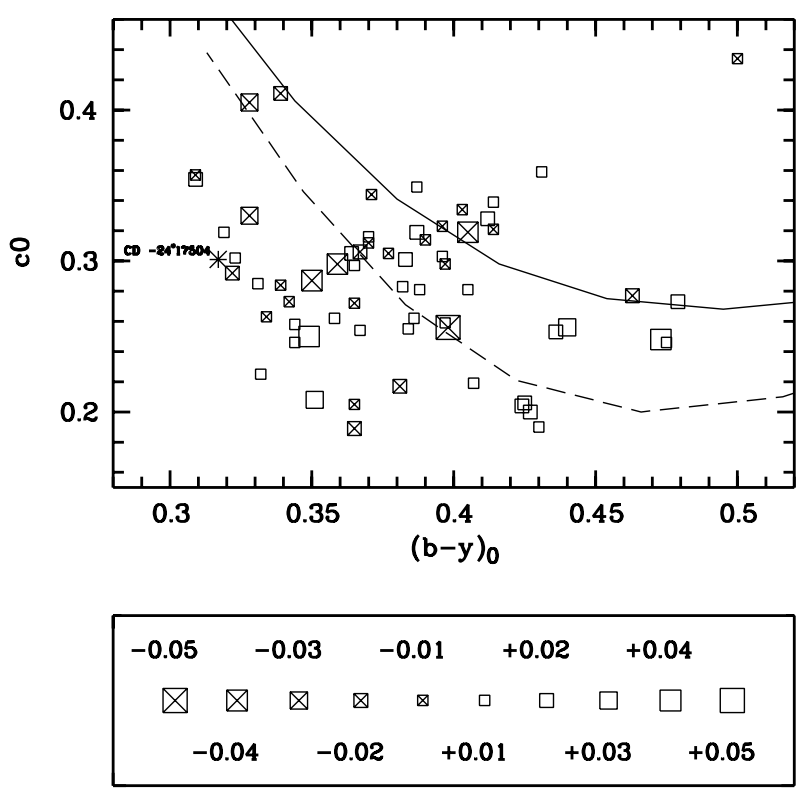

\section{scale of residuals}

Fig. 2. The residuals $(B-V)-$ FIT in the $\left((b-y)_{0}, c 0\right)$ plane. The stars have been divided into 10 bins 0.01 mag wide. The size of the symbol is largest for the stars in the bins $0.04<\mid(B-V)-$ FIT $\mid \leq 0.05 \mathrm{mag}$ and decreases to the minimum size for stars in the bins with $\mid(B-V)-$ FIT $\mid \leq 0.01$, as indicated in the scale plot shown below the figure. Negative residuals are shown with crossed squares, while positive residuals are shown with open squares. The star CD $-24^{\circ} 17504$ is drawn with an asterisk. The solid line represents the locus of points with $\log g=3.5$ for $[\mathrm{Fe} / \mathrm{H}]=-1.0$. The dashed line is the same but for $[\mathrm{Fe} / \mathrm{H}]=-2.5$ the calibrators and keep all the stars which had been rejected because either their reddening was too large or they lacked Strömgren photometry. Out of this sample we further selected only the stars with indices within the range of the calibration. The sample of comparison stars now consists of 129 stars, of which 71 have also Strömgren photometry. We start by comparing the reddening derived from Eq. (2) with that derived from the Schuster \& Nissen calibration through $E(B-V)=1.35 E(b-y)$. The result of the comparison is shown in panel a) of Fig. 3.

A clear outlier may be noticed (HD 7424), for which our reddening estimate is more than $0.1 \mathrm{mag}$ larger than that derived from Strömgren photometry. By dropping this star our sample eventually includes 70 comparison stars. The histogram of the difference $\left(E(B-V)_{\text {ours }}-\right.$ $\left.E(B-V)_{\mathrm{S}}\right)$ is shown in panel b) of Fig. 3. In Fig. 4, panels a) and b), we display the differences as a function of $[\mathrm{Fe} / \mathrm{H}]$ and $(B-V)$, no trend with either is apparent. The mean value of the difference is almost zero (0.002 mag) and the standard deviation is $0.025 \mathrm{mag}$. We note that if HD 7424 is kept in the sample only a slightly larger standard deviation of 0.028 mag would result. This shows that the reddening derived from our calibration is in good agreement with that derived from the Schuster \& Nissen calibration. The value of 0.025 may be regarded as an error estimate of the reddening derived through Eq. (2).

Next we compare our reddening with that reported by Beers et al. (1999), which is mostly based on the Burstein \& Heiles (1982) reddening maps (see Beers et al. 1999 for further details on their adopted reddening). The plot in which the reddenings are compared and the histogram of the differences $\left(E(B-V)_{\text {ours }}-E(B-V)_{\mathrm{B}}\right)$ are shown 

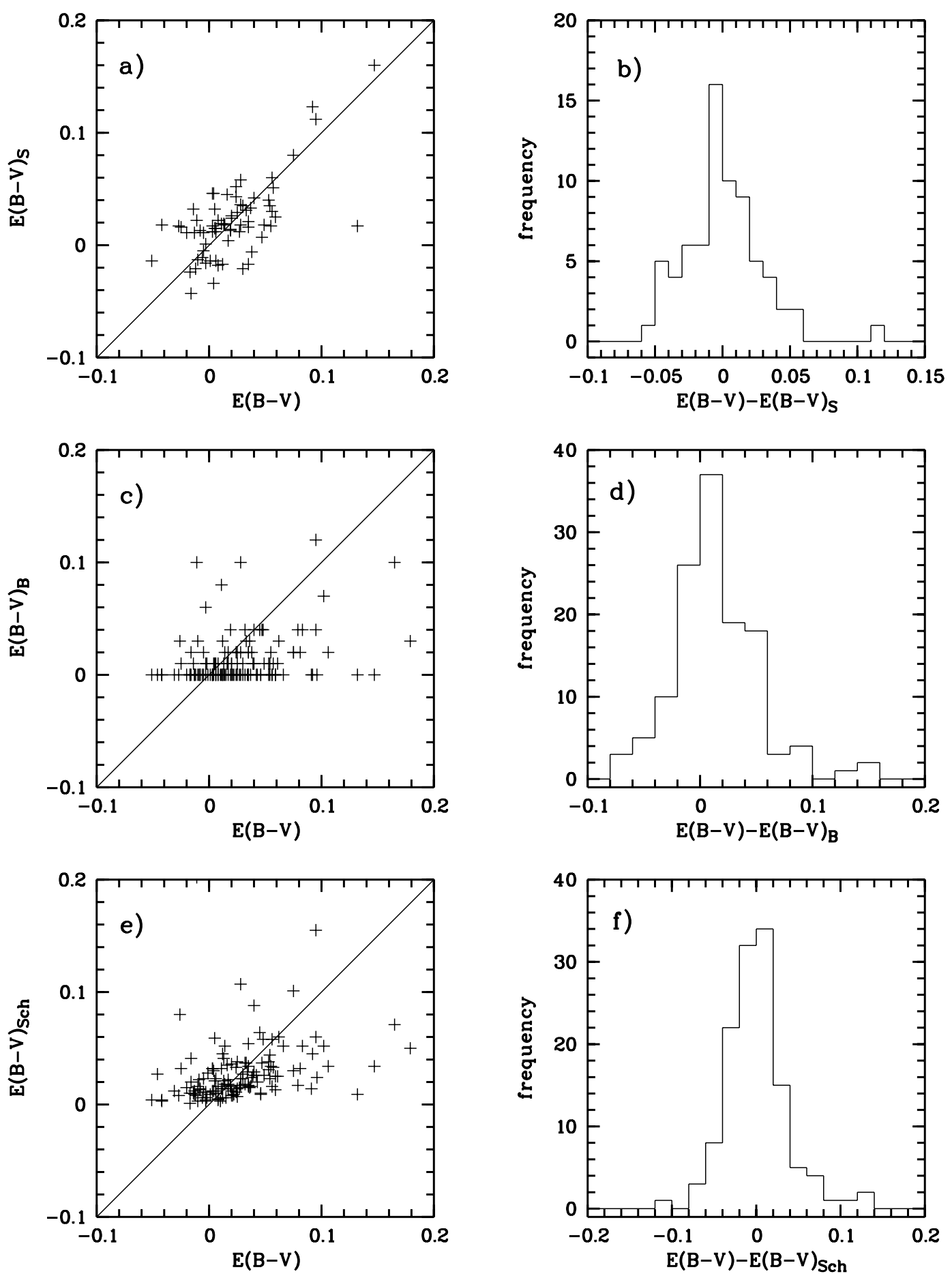

Fig. 3. Comparison of the reddening derived from Eq. (2) with that derived from Strömgren photometry (panels a) and b)), with that of Beers et al. (panels c) and d)) and with that derived from the maps of Schlegel et al. (panels e) and f))

in panels c) and d) of Fig. 3, respectively. There is an evident offset between the two reddening estimates as well as a tail with large differences. This is made up of three stars: HD 7424, already identified as an outlier in the comparison with reddening from Strömgren photometry; HD 161770 , for which Beers et al. (1999) give a zero reddening while we obtain 0.147 from Eq. (2) and 0.160 from the Schuster
\& Nissen calibration; G82-23 for which Beers et al. (1999) give 0.03 while we obtain 0.179 , where no Strömgren photometry is available for this star. The mean difference for the whole sample (129 stars) is 0.014 mag and the standard deviation is $0.040 \mathrm{mag}$. If we remove the three abovementioned stars from the sample the mean difference becomes $0.011 \mathrm{mag}$ and the standard deviation $0.034 \mathrm{mag}$. 


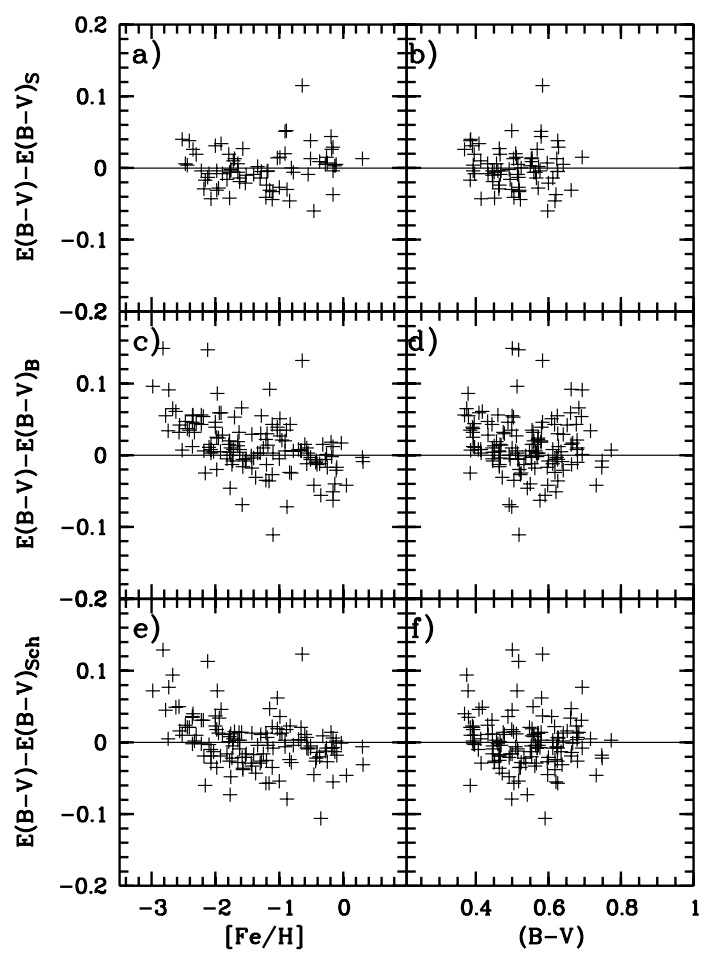

Fig. 4. Differences $E(B-V)_{\text {(our) }}-E(B-V)_{\text {(other) }}$ as a function of metallicity and $(B-V)$ colour. In panels a) and b) our reddening is compared to that derived from the Strömgren photometry; in panels c) and $\mathbf{d}$ ) to that given by Beers et al.; in panels $\mathbf{e}$ ) and f) to that derived form the maps of Schlegel et al.

In Fig. 4 panel c) we may notice a slight trend of the differences with metallicities, while no such trend appears with $(B-V)$ colour.

Finally, we compare our reddenings with those derived from the recent reddening maps of Schlegel et al. (1998). In order to obtain the $30 \%-50 \%$ reddening reduction recommended by Arce \& Goodman (1999) for the highly reddened stars we modify the reddenings above 0.10 mag as described in Bonifacio et al. $(2000)^{2}$. Several of our comparison stars are quite close and therefore within the dust layer. The reddening provided by the maps, refers instead to the full line of sight and should be applied as it stands only to extragalactic objects or to objects well above the dust layer. We take this into account by multiplying the reddening of the maps by a factor $[1-\exp (-|d \sin b| / h)]$, where $d$ is the star's distance $b$ its galactic latitude and $h$ the scale height of the dust layer, which we assumed to be 125 pc. The distances were taken from Beers et al. (1999). The plot of the reddening obtained from the Schlegel et al. (1998) maps versus our reddening is shown in panel e) of Fig. 3 and the histogram of the differences in panel $\mathrm{f})$. The mean value of the difference $\left(E(B-V)_{\text {our }}-E(B-V)_{\text {Sch }}\right)$

${ }^{2}$ For stars for which the reddening predicted by the Schlegel et al. (1998) maps $E(B-V)_{\mathrm{Sch}}>0.1$ Bonifacio et al. (2000) adopt $E(B-V)=0.10+0.65 \times\left[E(B-V)_{\mathrm{Sch}}-0.1\right]$.
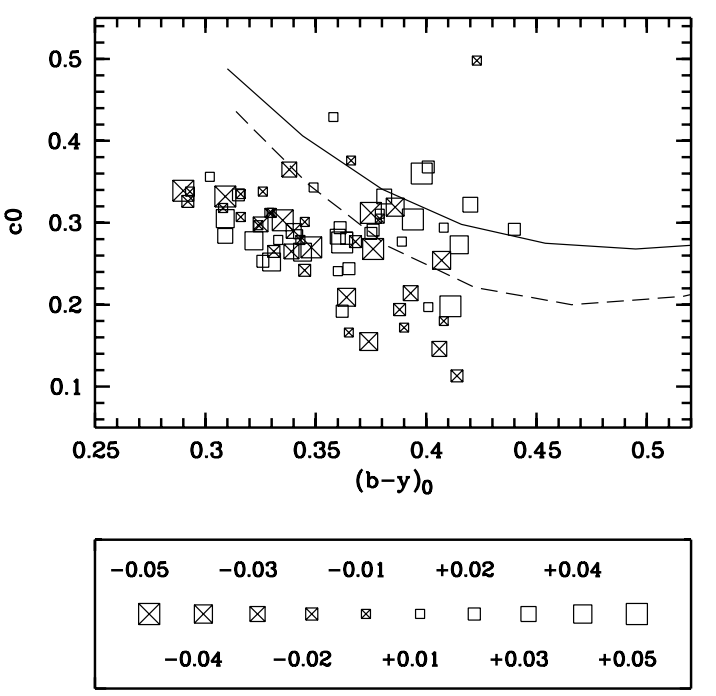

scale of residuals

Fig. 5. The residuals $E(B-V)-E(B-V)_{\mathrm{S}}$ in the $\left((b-y)_{0}, c 0\right)$ plane for the comparison stars. The stars have been divided into 10 bins 8 are 0.01 mag wide, one is $E(B-V)-E(B-V)_{\mathrm{S}}<$ -0.04 and one $E(B-V))-E(B-V)_{\mathrm{S}}>+0.04$. The rest of the symbols are as in Fig. 2

is $0.009 \mathrm{mag}$ and the standard deviation is $0.041 \mathrm{mag}$. Five stars out of the sample have absolute difference larger than $0.1 \mathrm{mag}$, namely G82-23, HD 7424 and HD 161770 have a difference $>0.1$, while G79-42 and G99-40 have a difference $<-0.1$. The reddening predicted by the Schlegel et al. maps for the latter two stars is very high, in spite of our reduction (0.411 for G79-42 and 0.707 for G99-40). If we treat these five stars as outliers and recompute both the mean and the standard deviation we obtain $0.013 \mathrm{mag}$ and 0.030 mag respectively. In Fig. 4 panel e), shows a slight dependence of the differences on metallicities, while panel f) shows no trend with $(B-V)$.

Our final check is on the possible dependence of the calibration on the luminosity of the stars. In Fig. 5 we show a box plot of the residuals in the $\left((b-y)_{0}, c 0\right)$ plane, similar to Fig. 2, but for the comparison stars, rather than for the calibrators. Among the comparison stars with Strömgren photometry there is only one giant seven subgiants and two horizontal branch stars, the rest are dwarfs or turnoff stars. Although the high luminosity stars are underrepresented there does not appear to be any obvious trend in the residuals with gravity.

From the above discussion we conclude that our reddening is comparable to that derived from Strömgren photometry through the Schuster \& Nissen calibration, while with respect to the reddening derived from the maps (either those of Schlegel et al. or those of Burstein \& Heiles) there is an offset of $\sim 0.01 \mathrm{mag}$, in the sense that the reddening predicted by Eq. (2) is higher than that predicted by the maps. The comparison also suggests that the accuracy of our reddening estimate is of the order of $0.03 \mathrm{mag}$, 
and therefore it is comparable to the reddening obtained from the Schuster \& Nissen calibration.

\section{Conclusions}

We derived an intrinsic colour calibration involving the broad band colours $(B-V)$ and $(U-B)$ and the line indices $H P 2$ and $K P$. Within its validity range the calibration may be used to derive the reddening $E(B-V)$ of individual stars with an accuracy of the order of 0.03 mag. We showed that the reddenings derived from our calibration are in good agreement with those derived from the Schuster \& Nissen (1989) calibration of Strömgren photometry. When compared to reddening determinations based on maps, either those of Burstein \& Heiles (1982) or those of Schlegel et al. (1998), our reddenings are systematically higher by about $0.01 \mathrm{mag}$.

The data base for $H P 2, K P,(B-V)$ and $(U-B)$ for HK survey stars is steadily increasing thanks to the efforts of Beers and collaborators and presently data for about 3000 stars are available. Our calibration may be used to derive reddenings for all these stars. Dereddened colours are necessary to determine the metallicity from the Beers et al. (1999) calibration and to derive a photometric distance. In the case of stars which will be observed spectroscopically at high resolution, dereddened colours will allow the determination of effective temperatures from colour-temperature calibrations.

Whenever uvby $\beta$ photometry is available the Schuster $\&$ Nissen calibration is the preferred way to determine the reddening of a star. However, when no Strömgren photometry is available, our calibration provides a useful way to estimate reddening with comparable accuracy.

Acknowledgements. We are grateful to Dr. A. Alonso, for carefully reviewing our paper and for suggesting many additions and improvements to its first version. This research made use of the SIMBAD data base operated at CDS, Strasbourg.

\section{References}

Alonso A., Arribas S., Martínez-Roger C., 1996, A\&A 313, 873 Alonso A., Arribas S., Martínez-Roger C., 1999, A\&AS 140, 261

Anthony-Twarog B.J., Sarajedini A., Twarog B.A., Beers T.C., 2000, AJ (in press) astro-ph/003115

Arce H.G., Goodman A.A., 1999, ApJ 512, L135

Beers T.C., 1999, in The Third Stromlo Symposium: The Galatic Halo, Gibson B.K., Axelrod T.S., Putman M.E. (eds.). San Francisco ASP 165, 206

Beers T.C., Preston G.W., Shectman S.A., 1985, AJ 90, 2089

Beers T.C., Preston G.W., Shectman S.A., 1992, AJ 103, 1987

Beers T.C., Rossi S., Norris J.E., Ryan S.G., Shefler T., 1999, AJ 117, 981

Bevington P.R., Robinson D.K., 1992, "Data Reduction and Error Analysis for the Physical Sciences" 2nd ed. WCB/McGraw-Hill

Bonifacio P., Monai S., Beers T.C., 2000, AJ (in press) astro-ph/0006178

Burstein D., Heiles C., 1982, AJ 87, 1165

Crawford D.L., 1975, AJ 80, 955

Di Benedetto G.P., 1998, A\&A 339, 858

Doinidis S.P., Beers T.C., 1990, PASP 102, 1392

Doinidis S.P., Beers T.C., 1991, PASP 103, 973

Hauck B., Mermilliod M., 1998, A\&AS 129, 431

Mermilliod J.C., Hauck B., Mermilliod M., 1996, General Catalog of Photometric Data, http://obswww.unige.ch/gcpd/gcdp.html

Norris J.E., Ryan S.G., Beers T.C., 1999, ApJS 123, 639

Olsen E.H., 1983, A\&AS 54, 55

Preston G.W., Shectman S.A., Beers T.C., 1991, ApJS 76, 1001

Schlegel D.J., Finkbeiner D.P., Davis M., 1998, ApJ 500, 525

Schuster W.J., Nissen P.E., 1989, A\&A 221, 65

Schuster W.J., Nissen P.E., Parrao L., Beers T.C., Overgaard L.P., 1996, A\&AS 117, 317 